1999-8

\title{
Nonlinear Robust Missile Autopilot Design Using Successive Galerkin Approximation
}

Timothy McLain

Mechanical Engineering Department, Brigham Young University, mclain@byu.edu

Randal W. Beard

Department of Electrical and Computer Engineering, Brigham Young University, beard@ee.byu.edu

Follow this and additional works at: https://scholarsarchive.byu.edu/facpub

Part of the Mechanical Engineering Commons

\section{Original Publication Citation}

McLain, T. and Beard, R. Nonlinear Robust Missile Autopilot Design Using Successive Galerkin Approximation, Proceedings of the AIAA Guidance, Navigation, and Control Conference, AIAA-1999-3997, August 1999, Portland, Oregon.

\section{BYU ScholarsArchive Citation}

McLain, Timothy and Beard, Randal W., "Nonlinear Robust Missile Autopilot Design Using Successive Galerkin Approximation" (1999). Faculty Publications. 1935.

https://scholarsarchive.byu.edu/facpub/1935 


\section{NONLINEAR ROBUST MISSILE AUTOPILOT DESIGN USING SUCCESSIVE GALERKIN APPROXIMATION}

\author{
Timothy W. McLain \\ Department of Mechanical Engineering \\ Brigham Young University \\ Provo, UT 84602 \\ tmclain@et. byu .edu
}

\author{
Randal W. Beard \\ Department of Electrical Engineering \\ Brigham Young University \\ Provo, UT 84602 \\ beard@ee.byu.edu
}

\begin{abstract}
The application of a new nonlinear robust control strategy to the design of missile autopilots is presented. The control approach described and demonstrated here is based upon the numerical solution of the Hamilton-Jacobi-Isaacs equation by Successive Galerkin Approximation. Using this approach, feedback controllers are computed by an iterative application of a numerical Galerkin-type PDE solver. Application of this approach to the design of a pitchaxis autopilot for a missile having uncertain pitch moment and lift force is described.
\end{abstract}

\section{INTRODUCTION}

The dynamics of guided launch vehicles are inherently nonlinear due to inertial coupling, gravitational forces, aerodynamic effects, and actuator limits. Though autopilot designs are typically based on linearized dynamic models, modern missile systems often operate in flight regimes where nonlinearities significantly affect dynamic response.

Much of the recently published missile control literature focuses on the application of linear robust control methods to linearized missile models. ${ }^{1-3}$ System nonlinearities are dealt with by using gain scheduled controllers or by treating nonlinear terms as uncertain. While the use of modern linear design methods has yielded improvements in control system performance and robustness, the use of nonlinear control methods may provide a means to improve control precision and widen stability boundaries when flight must be conducted at high angles or angular rates where nonlinear effects are most extreme.
The successive Galerkin approximation (SGA) has recently been developed for approximating the solution to the HJB and HJI equations in a form that is amenable to practical feedback control. ${ }^{10}$ The essential idea is to reduce the nonlinear HJB/HJI partial differential equation (PDE) to a convergent sequence of linear PDEs, and then to approximate the solution to each PDE via a global Galerkin approximation technique. The result is an implementable numerical algorithm that computes the coefficients needed for a nonlinear feedback control law that solves the nonlinear optimal $/ \mathcal{H}_{\infty}$ control problem. This technique has been successfully applied to a number of practical design problems. ${ }^{11-13}$ Of particular interest, the SGA algorithm has been used to synthesize a nonlinear optimal control for a missile autopilot system. ${ }^{14}$ The objective of this paper is to apply the SGA algorithm to synthesize a nonlinear $\mathcal{H}_{\infty}$ control for a similar missile autopilot system. 


\section{CONTROL APPROACH}

A wide variety of nonlinear dynamic systems (including the missile system considered here) can be modeled by equations of motion of the form

$$
\begin{array}{ll}
\dot{x}=f(x)+g(x) u+k(x) w & f(0)=0 \\
y=h(x) & h(0)=0
\end{array}
$$

where $x \in \mathbb{R}^{n}$ is the state of the system, $u \in \mathbb{R}^{m}$ is the control variable, $w \in \mathbb{R}^{q}$ is the disturbance signal and $y \in \mathbb{R}^{p}$ is the system output. This form can accommodate uncertainties in the parameters of the system with the $k(x) w$ term of Equation (1), where the elements of $w$ become the specific parametric uncertainties. The control objective of this paper is to develop control laws that attenuate the energy of the disturbance that is evident in the output through moderate amounts of control. This objective can be expressed mathematically as

$$
\int_{0}^{T}\left(\|y(t)\|^{2}+\|u(t)\|_{R}^{2}\right) d t \leq \gamma^{2} \int_{0}^{T}\|w(t)\|_{P}^{2} d t,
$$

for all $T>0$, and $\gamma>0$. If the above expression holds, the system is said to have $L_{2}$ gain, from the disturbance to the output and the control, less than $\gamma$ for all $w \in L_{2}(0, T)$ and $x(0)=0$.

The nonlinear $\mathcal{H}_{\infty}$ optimal control problem is to find the smallest $\gamma^{*}>0$ and an associated control $u^{*}$ such that the $L_{2}$ gain of the system, as defined by (3), is less than $\gamma^{*}$. The smaller the gain $\gamma^{*}$, the greater the attenuation of the disturbance that is achieved (i.e., the controlled system is less sensitive to variations in the system parameters). It has been shown $^{5}$ that a control law satisfying Equation (3) for system of Equation (1) can be found from the solution of the Hamilton-Jacobi-Isaacs (HJI) equation for $V_{\gamma} \geq 0$ :

$$
\begin{aligned}
& \frac{\partial V_{\gamma}^{T}}{\partial x} f+h^{T} h \\
& +\frac{1}{4} \frac{\partial V_{\gamma}^{T}}{\partial x}\left(\frac{1}{2 \gamma^{2}} k P^{-1} k^{T}-g R^{-1} g^{T}\right) \frac{\partial V_{\gamma}}{\partial x}=0
\end{aligned}
$$

where $V_{\gamma}(0)=0$ is a boundary condition. From the solution of the HJI equation for $V_{\gamma}$, the corresponding control $u_{\gamma}$ and disturbance $w_{\gamma}$ can be determined

$$
\begin{aligned}
u_{\gamma}(x) & =-\frac{1}{2} R^{-1} g^{T}(x) \frac{\partial V_{\gamma}}{\partial x} \\
w_{\gamma}(x) & =-\frac{1}{2 \gamma^{2}} P^{-1} k^{T}(x) \frac{\partial V_{\gamma}}{\partial x}
\end{aligned}
$$

which result in a closed-loop system having $L_{2}$ gain less than or equal to $\gamma$. Solving the nonlinear $\mathcal{H}_{\infty}$ control problem requires finding the smallest possible $\gamma^{*}$ for which Equation (4) has a solution and then solving Equation (4) for $V_{\gamma^{*}}$ to find $u_{\gamma^{*}}$ and $w_{\gamma^{*}}$. For nonlinear dynamical systems of practical interest, solution of the HJI equation is typically impossible analytically and very difficult numerically.

The HJI solution strategy followed here is based on the integration of two numerical strategies, successive approximation and Galerkin's method. ${ }^{10}$ Note that the HJI equation (4) can be written as

$$
\begin{aligned}
\frac{\partial V_{\gamma}^{T}}{\partial x}\left(f+g u_{\gamma}+k w_{\gamma}\right)+h^{T} h & \\
& +\left\|u_{\gamma}\right\|_{R}^{2}-\gamma^{2}\left\|w_{\gamma}\right\|_{P}^{2}=0
\end{aligned}
$$

where $u_{\gamma}$ and $w_{\gamma}$ are defined in Equations (5) and (6) respectively. By writing the HJI equation in this form, it can be seen that the nonlinearity in the HJI equation comes from the dependence of $u_{\gamma}$ and $w_{\gamma}$ on the value function $V_{\gamma}$. It is also evident from Equations (5), (6), and (7) that the HJI equation can be reduced to a sequence of linear partial differential equations by performing two simultaneous iterations of successive approximation. The successive approximation approach taken here, which is outlined by Algorithm 1, is to start with a known control that is stabilizing over a bounded domain $\Omega$ of the state space and to first compute (by successive approximation) the worst-case disturbance corresponding to the initial control. A second successive approximation is then used to find the control which gives the best possible system response to the worst-case disturbance. These two successive approximation steps are carried out in an iterative fashion and for $\gamma>\gamma^{*}$ converge to a solution of the HJI equation.

\section{Algorithm 1 (HJI: Successive Approximation)}

Let $u^{(0)}$ be an initial stabilizing control law for the system $(1)(w \equiv 0)$ with stability region $\Omega$.

For $i=0$ to $\infty$

$$
\begin{aligned}
& \text { Set } w_{\gamma}^{(i, 0)} \equiv 0 . \\
& \text { For } j=0 \text { to } \infty \\
& \text { Solve for } V_{\gamma}^{(i, j)} \text { from: } \\
& \qquad \frac{\partial V_{\gamma}^{(i, j) T}}{\partial x}\left(f+g u_{\gamma}^{(i)}+k w_{\gamma}^{(i, j)}\right)+h^{T} h \\
& \quad+\left\|u_{\gamma}^{(i)}\right\|_{R}^{2}-\gamma^{2}\left\|w_{\gamma}^{(i, j)}\right\|_{P}^{2}=0 .
\end{aligned}
$$


Update the Disturbance:

$$
w_{\gamma}^{(i, j+1)}=\frac{1}{2 \gamma^{2}} P^{-1} k^{T} \frac{\partial V_{\gamma}^{(i, j)}}{\partial x} .
$$

\section{End}

\section{Update the Control:}

$$
u_{\gamma}^{(i+1)}=-\frac{1}{2} R^{-1} g^{T} \frac{\partial V_{\gamma}^{(i, \infty)}}{\partial x} .
$$

\section{End}

The nonlinear $\mathcal{H}_{\infty}$ control law is calculated by finding the smallest possible $\gamma$ for which the successive approximation algorithm converges. In practice, the minimum $\gamma$ is approximated by embedding Algorithm 1 in a bisection search algorithm to find the smallest value of $\gamma$ for which the algorithm converges.

This successive approximation algorithm has an interpretation corresponding to the minimax problem of game theory, which has been associated with the $H_{\infty}$ control paradigm. ${ }^{6}$ The inner loop updates the disturbance variable for a given control law, so that $w_{\gamma}^{(i, \infty)}$ is the worst possible disturbance for the control $u_{\gamma}^{(i)}$. The outer loop updates the control law to give the best possible performance for a given worst case disturbance. Ultimately, the algorithm finds the worst possible disturbance for the system and the best possible control to mitigate the effects of the disturbance on the output of the system.

The key to the successive approximation algorithm is that the nonlinear HJI partial differential equation has been reduced to a sequence of linear partial differential equations given by Equation (8) which is called the Generalized Hamilton-JacobiIsaacs (GHJI) equation. Execution of Algorithm 1 requires the repeated numerical solution of the GHJI equation, which because it is linear is more easily done than the solution of the HJI equation. To solve the GHJI equation, a computational Galerkin method is used. ${ }^{15}$ Galerkin's method first assumes that the value function $V_{\gamma}^{(i, j)}$ can be written as an infinite series of known basis functions $\left\{\phi_{\ell}(x)\right\}_{\ell=1}^{\infty}$ that are continuous and defined everywhere on $\Omega$, i.e.,

$$
V_{\gamma}^{(i, j)}(x)=\sum_{\ell=1}^{\infty} c_{\ell}^{(i, j)} \phi_{\ell}(x)
$$

The unknown coefficients $c_{\ell}^{(i, j)}$ are found using the Galerkin solution strategy. As a practical issue, an infinite number of terms cannot be used to express
$V_{\gamma}^{(i, j)}$, so an approximation to the assumed solution having the desired degree of accuracy can be formed by considering the first $N$ terms of the infinite series:

$$
V_{\gamma, N}^{(i, j)}(x)=\sum_{\ell=1}^{N} c_{\ell}^{(i, j)} \phi_{\ell}(x) .
$$

By substituting Equation 11 into Equation 8, an expression for the error $e^{(i, j)}$, which results from approximating $V_{\gamma}^{(i, j)}$ with $V_{\gamma, N}^{(i, j)}$, is obtained:

$$
\begin{aligned}
e^{(i, j)}(x)= & \left(\sum_{\ell=1}^{N} c_{\ell}^{(i, j)} \frac{\partial \phi_{l}^{T}}{\partial x}\right)\left[f+g u_{\gamma}^{(i)}+k w_{\gamma}^{(i, j)}\right] \\
& +h^{T} h+\left\|u_{\gamma}^{(i)}\right\|_{R}^{2}-\gamma^{2}\left\|w_{\gamma}^{(i, j)}\right\|_{P}^{2} \cdot(12)
\end{aligned}
$$

Following Galerkin's method, the unknown coefficients $c_{\ell}^{(i, j)}$ can be found by taking the inner product of the error $e^{(i, j)}$ and the $N$ basis functions $\phi_{1}, \phi_{2}, \ldots, \phi_{N}$ on a closed and bounded set $\Omega$

$$
\left\langle e^{(i, j)}(x), \phi_{\ell}(x)\right\rangle_{\Omega}=0, \quad \ell=1 \ldots N
$$

where the inner product of two functions is defined in the following manner

$$
\langle f(x), g(x)\rangle_{\Omega} \triangleq \int_{\Omega} f(x) g(x) d x .
$$

The result of Equation (13) is $N$ linear equations in the $N$ unknown coefficients $c^{(i, j)}=$ $\left[c_{1}^{(i, j)}, c_{2}^{(i, j)}, \ldots, c_{N}^{(i, j)}\right]^{T}$ that can be solved as follows:

$$
c^{(i, j)}=\left(A_{1}+A_{2}^{(i)}+A_{3}^{(i, j)}\right)^{-1}\left(b_{1}+b_{2}^{(i)}+b_{3}^{(i, j)}\right)
$$

where

$$
\begin{aligned}
A_{1}= & {\left[\begin{array}{ccc}
\left\langle\frac{\partial \phi_{1}^{T}}{\partial x} f, \phi_{1}\right\rangle_{\Omega} & \cdots & \left\langle\frac{\partial \phi_{N}^{T}}{\partial x} f, \phi_{1}\right\rangle_{\Omega} \\
\vdots & \ddots & \vdots \\
\left\langle\frac{\partial \phi_{1}^{T}}{\partial x} f, \phi_{N}\right\rangle_{\Omega} & \cdots & \left\langle\frac{\partial \phi_{N}^{T}}{\partial x} f, \phi_{N}\right\rangle_{\Omega}
\end{array}\right] } \\
A_{2}^{(i)}= & {\left[\begin{array}{ccc}
\left\langle\frac{\partial \phi_{1}^{T}}{\partial x} g u_{\gamma}^{(i)}, \phi_{1}\right\rangle_{\Omega} & \cdots & \left\langle\frac{\partial \phi_{N}^{T}}{\partial x} g u_{\gamma}^{(i)}, \phi_{1}\right\rangle_{\Omega} \\
\vdots & \ddots & \vdots \\
\left\langle\frac{\partial \phi_{1}^{T}}{\partial x} g u_{\gamma}^{(i)}, \phi_{N}\right\rangle_{\Omega} & \cdots & \left\langle\frac{\partial \phi_{N}^{T}}{\partial x} g u_{\gamma}^{(i)}, \phi_{N}\right\rangle_{\Omega}
\end{array}\right] } \\
A_{3}^{(i, j)}= & {\left[\begin{array}{ccc}
\left\langle\frac{\partial \phi_{1}^{T}}{\partial x} k w_{\gamma}^{(i, j)}, \phi_{1}\right\rangle_{\Omega} & \cdots & \left\langle\frac{\partial \phi_{N}^{T}}{\partial x} k w_{\gamma}^{(i, j)}, \phi_{1}\right\rangle_{\Omega} \\
\vdots & \ddots & \vdots \\
\left\langle\frac{\partial \phi_{1}^{T}}{\partial x} k w_{\gamma}^{(i, j)}, \phi_{N}\right\rangle_{\Omega} & \cdots & \left\langle\frac{\partial \phi_{N}^{T}}{\partial x} k w_{\gamma}^{(i, j)}, \phi_{N}\right\rangle_{\Omega}
\end{array}\right] }
\end{aligned}
$$




$$
\begin{aligned}
b_{1}=-\left[\begin{array}{c}
\left\langle h^{T} h, \phi_{1}\right\rangle_{\Omega} \\
\vdots \\
\left\langle h^{T} h, \phi_{N}\right\rangle_{\Omega}
\end{array}\right] \\
b_{2}^{(i)}=-\left[\begin{array}{c}
\left\langle\left\|u_{\gamma}^{(i)}\right\|_{R}^{2}, \phi_{1}\right\rangle_{\Omega} \\
\vdots \\
\left\langle\left\|u_{\gamma}^{(i)}\right\|_{R}^{2}, \phi_{N}\right\rangle_{\Omega}
\end{array}\right] \\
b_{3}^{(i, j)}=\gamma^{2}\left[\begin{array}{c}
\left\langle\left\|w_{\gamma}^{(i, j)}\right\|_{P}^{2}, \phi_{1}\right\rangle_{\Omega} \\
\vdots \\
\left\langle\left\|w_{\gamma}^{(i, j)}\right\|_{P}^{2}, \phi_{N}\right\rangle_{\Omega}
\end{array}\right] .
\end{aligned}
$$

With the coefficients $c_{\ell}^{(i, j)}$ identified, a value for the partial derivative of the value function with respect to the state $\partial V_{\gamma}^{(i, j)} / \partial x$ associated with the $(i, j)^{t h}$ iteration can be calculated. This is used to calculate the worst-case disturbance $w_{\gamma}^{(i, j+1)}$, which will ultimately lead to the calculation of the control $u_{\gamma}^{(i+1)}$ associated with the $(i+1)^{t h}$ iteration:

$$
u_{\gamma}^{(i+1)}=-\frac{1}{2} R^{-1} g^{T}\left(\sum_{l=1}^{N} c_{l}^{(i, \infty)} \frac{\partial \phi_{l}}{\partial x}\right) .
$$

Although the matrices $A_{2}^{(i)}, A_{3}^{(i, j)}, b_{2}^{(i)}$, and $b_{3}^{(i, j)}$ change from one iteration to the next, their integrals need only be evaluated a single time. Values for $A_{2}^{(0)}$, $A_{3}^{(i, 0)}, b_{2}^{(0)}$, and $b_{3}^{(i, 0)}$ are calculated using the expressions above (remembering that $w_{\gamma}^{(i, 0)}=0$ ), while values for subsequent iterations can be calculated as follows:

$$
\begin{gathered}
A_{2}^{(i)}=-\frac{1}{2} \sum_{l=1}^{N} c_{l}^{(i-1, \infty)} M_{l} \\
b_{2}^{(i)}=-\frac{1}{4}\left[\begin{array}{c}
c^{(i-1, \infty) T} M_{1} c^{(i-1, \infty)} \\
c^{(i-1, \infty) T} M_{2} c^{(i-1, \infty)} \\
\vdots \\
c^{(i-1, \infty) T} M_{N} c^{(i-1, \infty)}
\end{array}\right]
\end{gathered}
$$

where

$$
\left\{M_{i}\right\}_{1}^{N}=\left[\begin{array}{ccc}
m_{i}(1,1) & \ldots & m_{i}(1, N) \\
\vdots & \ddots & \vdots \\
m_{i}(N, 1) & \ldots & m_{i}(N, N)
\end{array}\right]
$$

$$
\begin{gathered}
m_{i}(j, k)=\left\langle\frac{\partial \phi_{k}^{T}}{\partial x} g R^{-1} g^{T} \frac{\partial \phi_{i}}{\partial x}, \phi_{j}\right\rangle_{\Omega} . \\
A_{3}^{(i, j)}=\frac{1}{2 \gamma^{2}} \sum_{l=1}^{N} c_{l}^{(i, j-1)} S_{l} \\
b_{3}^{(i, j)}=\frac{1}{4 \gamma^{2}}\left[\begin{array}{c}
c^{(i, j-1) T} S_{1} c^{(i, j-1)} \\
c^{(i, j-1) T} S_{2} c^{(i, j-1)} \\
\vdots \\
c^{(i, j-1) T} S_{N} c^{(i, j-1)}
\end{array}\right]
\end{gathered}
$$

where

$$
\begin{gathered}
\left\{S_{i}\right\}_{1}^{N}=\left[\begin{array}{ccc}
s_{i}(1,1) & \ldots & s_{i}(1, N) \\
\vdots & \ddots & \vdots \\
s_{i}(N, 1) & \ldots & s_{i}(N, N)
\end{array}\right] \\
s_{i}(j, k)=\left\langle\frac{\partial \phi_{k}^{T}}{\partial x} k P^{-1} k^{T} \frac{\partial \phi_{i}}{\partial x}, \phi_{j}\right\rangle_{\Omega} .
\end{gathered}
$$

Calculation of the basis function coefficients as outlined above can be computationally demanding. It should be noted that these computations are part of the synthesis procedure and are carried out prior to implementation of the control. The computations that are carried out as part of the implementation are relatively simple and are easily done in real time with hardware having modest computational performance.

\section{MISSILE SYSTEM MODEL}

This paper addresses the design of an autopilot for the pitch-plane motion of a tail-controlled missile system. The mathematical model for the system under consideration is based upon the model originally presented by Reichert ${ }^{2,16}$ for a missile traveling at Mach 3 at an altitude of 20,000 ft. Figure 1 shows a schematic representation of the missile and the coordinates used to represent its motion.

The equations of motion which describe the dynamics of the missile are given by

$$
\begin{aligned}
\dot{q} & =\frac{M_{y}}{I_{y}} \\
\dot{\alpha} & =\frac{\cos ^{2}(\alpha)}{m U} F_{z}+q \\
\ddot{\delta} & =-2 \zeta \omega_{n} \dot{\delta}-\omega_{n}^{2} \delta+\omega_{n}^{2} \delta_{c}
\end{aligned}
$$




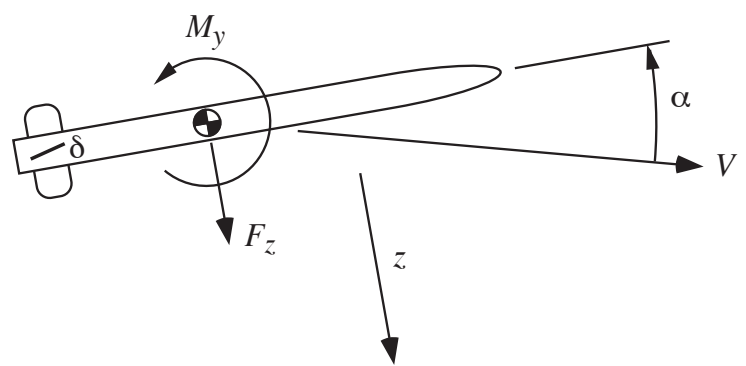

Figure 1: Missile System Pitch-Plane Schematic

$$
\begin{array}{cl}
\text { where } & =\text { pitch rate, deg/s } \\
q & =\text { angle of attack, deg } \\
\alpha & =\text { tail fin deflection, deg } \\
\delta & =C_{m}(\alpha, \delta) Q S d, \mathrm{ft}-1 \mathrm{~b} \\
\delta_{c} & =\text { commanded tail fin deflection, deg } \\
M_{y} & =C_{n}(\alpha, \delta) Q S, \mathrm{~b} \\
F_{z} & =C_{n} \\
I_{y} & =\text { pitch moment of inertia, } 182.5 \mathrm{slug}-\mathrm{ft}^{2} \\
m & =\text { mass of missile, } 13.98 \mathrm{slug} \\
U & =\text { longitudinal velocity, } V \cos (\alpha), \mathrm{ft} / \mathrm{s} \\
\zeta & =\text { fin actuator damping ratio, } 1.4 \\
\omega_{n} & =\text { fin actuator natural frequency, } 5 \mathrm{~Hz} \\
M & =\text { Mach number, } 3 \\
Q & =\text { dynamic pressure, } 0.7 \rho M^{2}, 1 \mathrm{lb} / \mathrm{ft}^{2} \\
S & =\text { reference area, } 0.44 \mathrm{ft} \\
d & =\text { diameter, } 0.75 \mathrm{ft} \\
\rho & =\text { static pressure at } 20,000 \mathrm{ft}, 973.3 \mathrm{lb} / \mathrm{ft}^{2} \\
s_{s} & =\text { speed of sound at } 20,000 \mathrm{ft}, 1036.4 \mathrm{ft} / \mathrm{s} \\
V & =\text { velocity of missile, } M s_{s}, \mathrm{ft} / \mathrm{s} .
\end{array}
$$

The aerodynamic coefficients, $C_{n}$ and $C_{m}$ can be represented by the following polynomial functions of $\alpha$ and $\delta$ :

$$
\begin{aligned}
C_{n} & =a_{1} \alpha^{3}+a_{2} \alpha|\alpha|+a_{3} \alpha+a_{4} \delta \\
a_{1} & =.000103 \\
a_{2} & =-.00945 \\
a_{3} & =-.170 \\
a_{4} & =-.034 \\
C_{m} & =b_{1} \alpha^{3}+b_{2} \alpha|\alpha|+b_{3} \alpha+b_{4} \delta \\
b_{1} & =.000215 \\
b_{2} & =-.0195 \\
b_{3} & =.051 \\
b_{4} & =-.206 .
\end{aligned}
$$

The robust controller synthesis presented here considers uncertainty in the pitching moment $M_{y}$ and the lift force $F_{z}$ as disturbances to the system. In practice, the parameters that describe $M_{y}$ and $F_{z}$ are uncertain because of the difficulty in accurately modeling the aerodynamic forces and moments for widely ranging values of $\alpha$ and $\delta$. Furthermore, variations from the nominal value of $M=3$ can result in significant changes in the aerodynamic forces and moments that are acting on the missile.

If the nominal values for pitching moment and lift force, $\hat{M}_{y}$ and $\hat{F}_{z}$, are defined as

$$
\begin{aligned}
\hat{M}_{y} & =C_{m} Q S d \\
\hat{F}_{z} & =C_{n} Q S
\end{aligned}
$$

and the actual values for pitching moment and lift force are represented as

$$
\begin{aligned}
M_{y} & =(1+\mu) \hat{M}_{y} \\
F_{z} & =(1+\nu) \hat{F}_{z},
\end{aligned}
$$

then the pitch rate and angle of attack equations of motion can be written as

$$
\begin{aligned}
& \dot{q}=\frac{C_{m} Q S d}{I_{y}}+\frac{C_{m} Q S d}{I_{y}} \mu \\
& \dot{\alpha}=\frac{C_{n} Q S \cos ^{2}(\alpha)}{m U}+q+\frac{C_{n} Q S \cos ^{2}(\alpha)}{m U} \nu,
\end{aligned}
$$

where $w=[\mu \nu]^{T}$ are the parametric uncertainties that act as disturbances to the system.

For this system, the output of interest is the angle of attack of the missile, $\alpha$. The control synthesis algorithm developed above provides state feedback to regulate the states to zero. The missile autopilot design objective is to control the angle of attack to a non-zero reference value. To do so, the pitch rate $q$, the fin deflection angle $\delta$, and the control $\delta_{c}$ must be non-zero in steady state. For the SGA algorithm to be applicable, a change of variables must take place.

With the missile operating at the desired steadystate angle of attack $\alpha_{d}$, the values of the states, $\left[q_{s s} \alpha_{s s} \dot{\delta}_{s s} \delta_{s s}\right]^{T}$, and the control, $\delta_{c, s s}$, required to for this angle of attack to be maintained can be found by solving the nonlinear system of equations resulting from the state equations and output equation when the state derivatives are set to zero

$$
\begin{aligned}
0 & =\frac{M_{y, s s}}{I_{y}} \\
0 & =\frac{\cos ^{2}\left(\alpha_{s s}\right)}{m U} F_{z, s s}+q_{s s} \\
0 & =-\omega_{n}^{2} \delta_{s s}+\omega_{n}^{2} \delta_{c, s s} \\
\alpha_{d} & =\alpha_{s s}
\end{aligned}
$$

with $M_{y, s s}$ and $F_{z, s s}$ determined from the relations for $M_{y}$ and $F_{z}$ defined above. 
By performing the change of variables

$$
x=\left[\begin{array}{c}
x_{1} \\
x_{2} \\
x_{3} \\
x_{4}
\end{array}\right]=\left[\begin{array}{c}
q-q_{s s} \\
\alpha-\alpha_{s s} \\
\dot{\delta}-\dot{\delta}_{s s} \\
\delta-\delta_{s s}
\end{array}\right]
$$

for the states and $u=\delta_{c}-\delta_{c, s s}$ for the control on the equations of motion, a new set of state equations results where a stabilizing control will cause $\alpha \rightarrow \alpha_{d}$, $x \rightarrow 0$, and $u \rightarrow 0$ as $t \rightarrow \infty$, thereby enabling the application of the SGA algorithm.

For the purpose of the control synthesis, the output was selected to be $y=\left[0.1 x_{1} x_{2}\right]^{T}$ This ensured that the regulation errors for $x_{1}$ and $x_{2}$ were considered in the formulation of the control. Even though the primary output of interest is $x_{2}=\alpha-\alpha_{s s}$, including other states in the output provides the designer with flexibility in modifying the behavior of the closed-loop system. From the model presented here, the functions $f(x), g(x), k(x)$, and $h(x)$ can be easily identified and the equations of motion can be placed in the form of Equation (1).

\section{CONTROL SYNTHESIS AND IMPLEMENTATION}

With the model described above, a robust feedback control law can be synthesized based on the approach presented previously. The functions $f(x)$, $g(x), k(x)$, and $h(x)$ are determined directly from the dynamic model of the system. The weighting matrices on the control cost $R$ and the disturbance cost $P$ are determined by the designer. In this case, $R$ is simply a scalar, since there is only one control input to the system $\delta_{c}$, while $P$ is a $2 \times 2$ matrix due to the two parametric uncertainties $\mu$ and $\nu$. For the results presented, both $R$ and $P$ were identity.

The domain of possible values for the states is determined both by the physical capabilities of the system and the likely deviation of the states from their nominal value of zero. For the results presented here, $\Omega$ was defined to be

$$
\begin{aligned}
-150 \mathrm{deg} / \mathrm{s} & \leq x_{1} \leq 150 \mathrm{deg} / \mathrm{s} \\
-20 \mathrm{deg} & \leq x_{2} \leq 20 \mathrm{deg} \\
-800 \mathrm{deg} / \mathrm{s} & \leq x_{3} \leq 800 \mathrm{deg} / \mathrm{s} \\
-20 \mathrm{deg} & \leq x_{4} \leq 20 \mathrm{deg} .
\end{aligned}
$$

Proper selection of the basis functions is a critical part in the design of controllers using SGA. The basis functions used determine not only the accuracy of the Galerkin approximation, but also the functions of the states from which the control law is calculated (see Equation 15). The selection of the basis functions also has implications on the operation of the SGA algorithm. If the basis functions do not approximate the value function $V_{\gamma}^{(i, j)}(x)$ with sufficient accuracy, the algorithm will fail to converge. For this problem, a second-order set of polynomial basis functions was used:

$$
\begin{array}{r}
\left\{\phi_{j}\right\}_{j=1}^{10}=\left\{x_{1}^{2}, x_{1} x_{2}, x_{2}^{2}, x_{1} x_{3}, x_{2} x_{3}, x_{3}^{2},\right. \\
\left.x_{1} x_{4}, x_{2} x_{4}, x_{3} x_{4}, x_{4}^{2},\right\} .
\end{array}
$$

This set of basis functions results in a full-state feedback control law.

The initial stabilizing control was designed by linearizing the equations of motion about the desired angle of attack and using pole placement. For this problem, the following initial control was used:

$$
u_{\gamma}^{(0)}=.480 x_{1}-2.82 x_{2}-.0732 x_{3}-3.77 x_{4} .
$$

\section{RESULTS}

Using the SGA synthesis algorithm, a nonlinear $\mathcal{H}_{\infty}$ control law was computed for the pitch axis of the missile system. As a demonstration of the robustness of the control achieved, the pitch moment and lift force were allowed to increase and decrease by a factor of two from their nominal values. Such large variations are extreme, but could occur due to significant changes in the Mach number $\left(M_{y}\right.$ and $F_{z}$ are functions of $M^{2}$ and $M^{3}$ ) or damage to the aerodynamic surfaces of the missile. These large variations serve to demonstrate the robustness of the control achieved. In Figure 2, state trajectories are plotted for the following perturbation cases: $[\mu=0, \nu=0],[\mu=1, \nu=1],[\mu=-.5, \nu=-.5]$, $[\mu=-.5, \nu=1]$, and $[\mu=1, \nu=-.5]$.

In each case, the missile was given zero initial conditions and then given a 15 degree angle of attack command . It can be seen from the response of the system under differing perturbation conditions that variations in $M_{y}$ and $F_{z}$ do affect the performance of the system. Figure 2 shows that the steady-state response of the system is most sensitive to variations in $F_{z}$ (changes in $\nu$ ), while variations in $M_{z}$ (changes in $\mu$ ) have an effect on the stability and transient response of the missile.

The stabilizing control law $u_{\gamma}^{(0)}$ used to initiate the SGA algorithm was a high-bandwidth full-state feedback controller designed using pole-placement. 

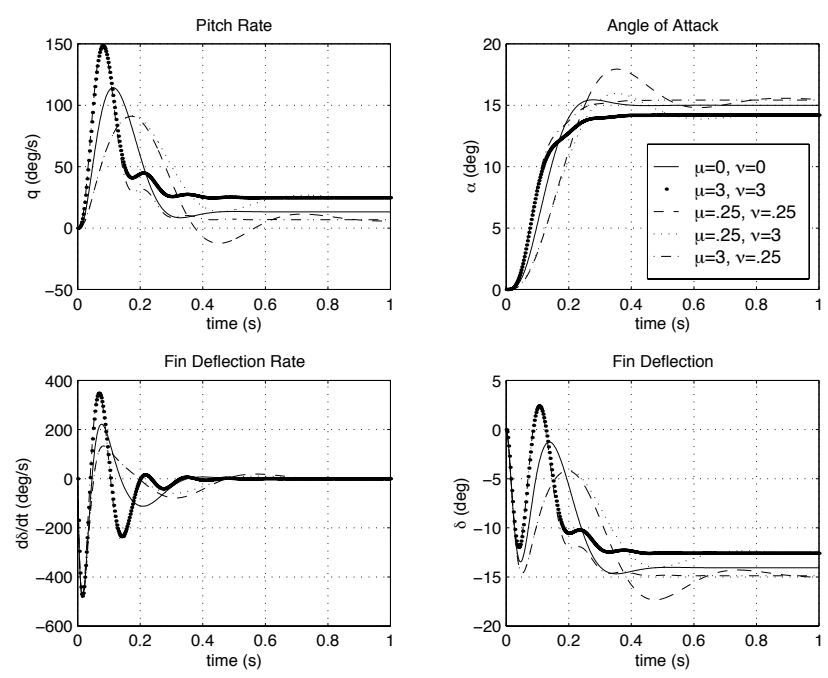

Figure 2: System Response with Parametric Variation

When tested with variations in $M_{y}$ and $F_{z}$, this controller exhibited similar robustness characteristics to the robust SGA control law developed above. Initially, this cast some doubt onto the correctness of the results obtained from the SGA synthesis algorithm. Examination of the algorithm provides insight into the results obtained. Referring to Algorithm 1 , it can be seen that the inner loop calculates the worst possible disturbance (parameter variation) for a given control. The outer loop subsequently calculates the best possible control for this worst-case disturbance. These operations are carried out iteratively, using successively smaller values of $\gamma$ until the smallest $\gamma$ is found that results in convergence of the algorithm.

Figure 3 shows the closed-loop response of the system with the SGA robust controller and the worstcase parameter variations acting. Note from Equation 9 that $w_{\gamma}$ (and hence the parametric variations $\mu$ and $\nu$ ) is a function of the states of the system. It can be seen that the response of the system has been degraded slightly by the parametric variations. Notice that the worst-case variation in $\mu$ is quite significant, while the worst-case variation in $\nu$ is essentially zero. This result reinforces the results of Figure 2 which also indicate that the stablity of the system is most affected by changes in $\mu$.

When the pole-placement controller was tested with the same parametric variations shown in Figure 3, satisfactory results were also obtained. To better determine a measure of the robustness of the two controllers, the following cost function was com-
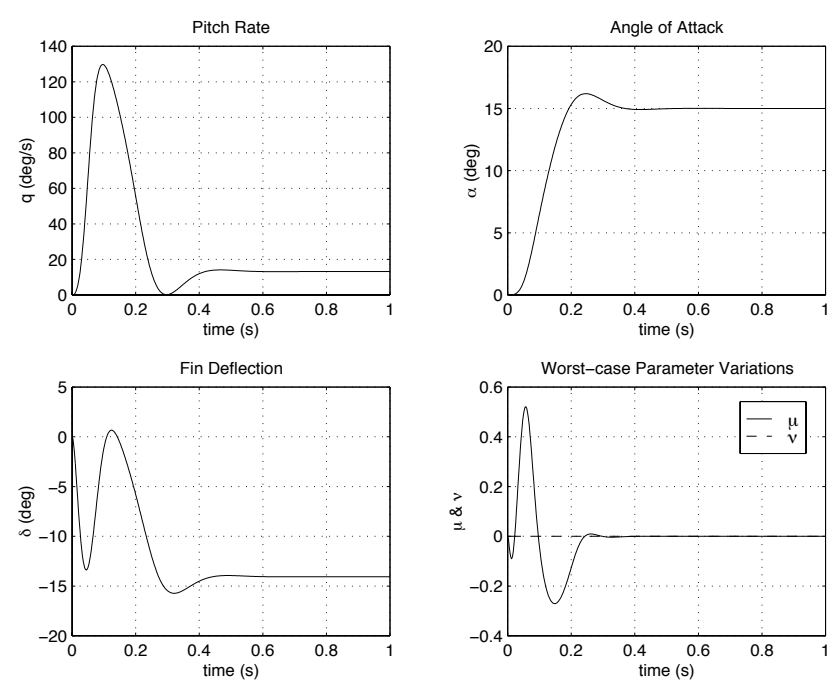

Figure 3: System Response with Worst-case Parametric Variations

puted for the system with both controllers:

$$
J=\int_{0}^{1}\left(\|y(t)\|^{2}+\|u(t)\|_{R}^{2}\right) d t .
$$

For the SGA robust design, the cost was calculated to be 132.9. For the pole-placement design, the cost was 182.6. This comparison demonstrates that for the two designs (which both employ linear full-state feedback), the SGA robust design results in greater attenuation of the disturbance, as manifest in the output and the control, than the traditional design.

A possible explanation for achieving only modest improvements in robustness with the SGA approach could be that modeling the parametric uncertainty as an $L_{2}$ disturbance is overly conservative. In practice, uncertainties in the parameters are most likely constant or slowly changing and in general do not require a characterization as broad as $L_{2}$ represents. By optimizing over all possible $L_{2}$ parameter variations, robustness to variations at lower, more realistic bandwidths is possibly compromised.

It should also be noted that the SGA design is optimal with respect to the basis functions used in the approximation. It is likely that a different set of basis functions could be chosen that would result in a better approximation of $V_{\gamma}^{(i, j)}$, and hence a more robust design.

\section{CONCLUSIONS}

The Successive Galerkin Approximation algorithm for numerically solving the Hamilton-Jacobi- 
Isaacs equation has been presented. Using this synthesis algorithm, a nonlinear robust controller has been designed for the pitch-axis autopilot of an agile missile. These feedback controllers were designed taking into account the full nonlinear dynamics of the missile system. Simulation results demonstrated improvements in robustness over linear full-state feedback designs.

\section{REFERENCES}

[1] G. Gu, J. R. Cloutier, and G. Kim, "Gain scheduled missile autopilot design using observer-based $H_{\infty}$ control", in Proceedings of the American Control Conference, 1995, pp. 1951-1955.

[2] G. J. Balas, R. Lind, and A. Packard, "Optimally scaled $H_{\infty}$ full information control synthesis with real uncertainty", AIAA Journal of Guidance, Control, and Dynamics, vol. 19, no. 4, pp. 854-862, 1996.

[3] S.M. Yang and N.H. Huang, "Application of $H_{\infty}$ control to pitch autopilot of missiles", IEEE Transactions on Aerospace and Electronic Systems, vol. 32, no. 1, pp. 426-433, 1996.

[4] Frank L. Lewis, Optimal Control, John Wiley and Sons, New York, New York, 1986.

[5] A. J. van der Schaft, " $L_{2}$-gain analysis of nonlinear systems and nonlinear state feedback $H_{\infty}$ control", IEEE Transactions on Automatic Control, vol. 37, no. 6, pp. 770-784, June 1992.

[6] Tamer Basar and Pierre Bernhard, $H_{\infty^{-}}$ Optimal Control and Related Minimax Design Problems, Birkhaüser, 1995.

[7] W. L. Garrard, D. F. Enns, and S. A. Snell, "Nonlinear feedback control of highly manoeuvrable aircraft", International Journal of Control, vol. 56, no. 4, pp. 799-812, 1992.

[8] J. Huang and C.-F. Lin, "Numerical approach to computing nonlinear $H_{\infty}$ control laws", AIAA Journal of Guidance, Control, and Dynamics, vol. 18, no. 5, pp. 989-996, 1995.

[9] K. A. Wise and J. L. Sedwick, "Nonlinear $H_{\infty}$ optimal control for agile missiles", AIAA Journal of Guidance, Control, and Dynamics, vol. 19, no. 1, pp. 157-165, 1996.
[10] Randal W. Beard and Timothy W. McLain, "Successive Galerkin approximation algorithms for nonlinear optimal and robust control", International Journal of Control: Special Issue on Breakthroughs in the Control of Nonlinear Systems, vol. 71, no. 5, pp. 717-743, 1998.

[11] Timothy W. McLain and Randal W. Beard, "Nonlinear optimal control of a hydraulically actuated positioning system", in Proceedings of the ASME International Mechanical Engineering Congress and Exposition, Fluid Power Systems and Technology Division, Dallas, TX, November 1997.

[12] Timothy W. McLain and Randal W. Beard, "Nonlinear robust control of an electrohydraulic positioning system", in Proceedings of the ASME International Mechanical Engineering Congress and Exposition, Fluid Power Systems and Technology Division, Anaheim, CA, 1998.

[13] Timothy W. McLain, Christopher A. Bailey, and Randal W. Beard, "Synthesis and experimental testing of a nonlinear optimal tracking controller", in Proceedings of the American Control Conference, San, Diego, CA, 1999.

[14] Timothy W. McLain and Randal W. Beard, "Nonlinear optimal control design of a missile autopilot", in AIAA Guidance, Navigation and Control Conference, Boston, MA, 1998.

[15] C.A.J. Fletcher, Computational Galerkin Methods, Springer-Verlag, 1984.

[16] R. T. Reichert, "Robust autopilot design using $\mu$-synthesis", in Proceedings of the American Control Conference, 1990, pp. 2368-2373. 